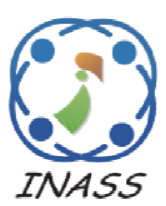

\title{
Deep Learning for Stock Market Index Price Movement Forecasting Using Improved Technical Analysis
}

\author{
Chinthakunta Manjunath ${ }^{1 *}$ \\ Balamurugan Marimuthu' \\ Bikramaditya Ghosh ${ }^{2}$ \\ ${ }^{1}$ Department of Computer Science and Engineering, CHRIST (Deemed to be University), Bengaluru, India \\ ${ }^{2}$ Department of Finance, RV Institute of Management, Bengaluru, India \\ * Corresponding author's Email: chinthakunta.manjunath@ christuniversity.in
}

\begin{abstract}
Equity market forecasting is difficult due to the high explosive nature of stock data and its impact on investor's stock investment and finance. The stock market serves as an indicator for forecasting the growth of the economy. Because of the nonlinear nature, it becomes a difficult job to predict the equity market. But the use of different methods of deep learning has become a vital source of prediction. These approaches employ time-series stock data for deep learning algorithm training and help to predict their future behavior. In this research, deep learning methods are evaluated on the India NIFTY 50 index, a benchmark Indian equity market, by performing a technical data augmentation approach. This paper presents a Recurrent Neural Network (RNN), Long Short Term Memory (LSTM), and the three variants of Gated Recurrent Unit (GRU) to analyze the model results. The proposed three GRU variants technique is evaluated on two sets of technical indicator datasets of the NIFTY 50 index (namely TA1 and TA2) and compared to the RNN and LSTM models. The experimental outcomes show that the GRU variant1 (GRU1) with TA1 provided the lowest value of Mean Square Error (MSE=0.023) and Root Mean Square Error (RMSE= 0.152) compared with existing methods. In conclusion, the NIFTY 50 index experiments with technical indicator datasetTA1 were more efficient by GRU. Hence, TA1 can be used to construct a robust predictive model in forecasting the stock index movements.
\end{abstract}

Keywords: Deep learning method, Gated recurrent unit, Relative volatility index, Ease of movement, Reynolds number, Market forecasting.

\section{Introduction}

Equity market forecasting is one of the significant areas and growing research in economic time series forecasting. However, equity market features such as noisy and non-stationary phenomena make forecasting face challenges [1, 2]. Several methods are used to forecast stock prices to examine previous price movements in the equity market. Linear and nonlinear methods like Artificial Neural Networks (ANN) and autoregressive methods predict the equity returns [3]. The equity market forecasting attained considerable attention in both theoretical and application views. The abundance of data sources offers the ability to comprehensively predict the stock market value and make the price forecast more precise than ever [4].
The conventional equity market forecasting methods consider historical price-related data. Some mechanisms try to discover financial news to improve the forecast based on technical analysis [5]. Social Networks such as Twitter and Facebook data were analyzed by various scientists for equity market forecasting [6]. The initiation of big data and the improvement of expert system technologies pave the way to improve stock market prediction with unstructured data. However, the efficiency of stock market forecasting still needs to be improved [7]. The practice of sentiment and attention indicators for equity market forecasting using machine learning helps to enhance efficiency. Twitter features and Kalman Filter (KF) were helpful in predict some stock's profits, and the usage of this model is the active research topic [8]. Moreover, the latest academic literature proves the widespread prediction 
of time series based on neural networks. The ANN method shows a compelling performance in analyzing the nonlinear nature of the equity market data and has superior performance in equity market forecasting [9].The deep learning methods study the nonlinear relationships in the features that provide better results. These methods consist of the innovative generation's artificial neural networks to overcome traditional problems like overfitting and vanishing gradient problems $[10,11]$. The closing price of Standard \& Poor's (S\&P) Bombay Stock Exchange (BSE) 100 Index in the Indian Capital Market is forecasted using Artificial Neural Network (ANN) and technical analysis [12].

To forecast CNX Nifty closing on certain specified time zones, the researcher used a neural network as a predictive tool. The Neural Network in this market is an excellent method for identification or prediction [13]. Extraction of features is an integral part of the prediction process of the stock index. Better market characteristics always make for better forecasts. Technical analysis is carried out mainly to extract attributes from the equity market information [14]. The correlation between the features of the technical analysis and stock index value direction is frequently explored through the use of machine learning methods like K-nearest neighbor $(\mathrm{kNN})$, ANN, Support Vector Machine (SVM), and Random Forest (RF) [15]. A financial version of the Reynolds number is used to classify potential volatile fluctuations in the CNX Nifty Index in the financial market. The relative volatility index (RVI) and ease of movement (EMV) are computed for confirmation of the Reynolds Number (RN) in this work [16].

Much research has recently been conducted to forecast equity market actions using deep learning and technical analysis, which has been efficient for the financial market domain. However, there are numerous technical indicators, and using accurate indicators can sometimes improve forecasting accuracy. As a result, in this work, a technical data augmentation for equity market index forecasting with deep learning is performed to improve forecasting accuracy.

In this research, we propose three gated variants of the GRU, which has proven to be highly efficient in financial sequence data forecasting problems for predicting the direction of the equity market. We used two distinct technical feature sets, TA1 and TA2. In our model, the feature set (TA1) employs eight technical indicators (as illustrated in Table 2), while the feature set (TA2) employs four technical indicators (as described in Table 3). The following is the procedure for developing this forecasting system: First, using the NIFTY 50 index OHLC and volume variables, technical data augmentation is performed to generate two types of input sets (TA1 or TA2), and then these TA1 and TA2 features are passed on to the proposed models.

The following are the summaries of our contributions:

- A popular deep learning model called GRU is adopted to forecast the direction of the NIFTY 50 index, financial time series value.

- Three gate variants of the GRU model are used to predict India NIFTY 50 index closing price movement by performing a technical data augmentation approach, which can enhance the model's overall predictive capability.

- The developed GRU variants are analyzed on two sets of technical indicator features, namely, TA1 and TA2 of the NIFTY 50 index and the standard LSTM and RNN methods. These proposed three variants of GRU will derive additional conceptual and invariant features.

- Two sets of technical parameters (namely TA1 or TA2) are used as features to make predictions, and TA 1 was found to be more efficient by GRU. Hence, TA1 can be used to construct a robust predictive model.

The paper is formulated as, the literature survey of recent stock market forecasting is given in section 2 , an overview of traditional RNN, LSTM and the proposed three variants of GRU is explored in section 3 , the experimental findings are addressed in section 4 and conclusion is given in section 5 .

The NIFTY 50 index's daily price data and two sets of technical indicators are used as empirical samples to authenticate the model's legitimacy proposed in this article. Two benchmark models are chosen, namely, RNN and LSTM, respectively.

\section{Literature survey}

Many related studies in the financial field have used various computational intelligence methods and technical analysis to solve individual stock price and stock market forecasting problems over the years. This section included a review of the literature on these methods.

A data augmentation method named ModAugNet is used to solve the overfitting problem in LSTM and is evaluated with American and Korean stock market exchange data for forecasting the index. The ModAugNet model shows the effective performance in the stock market forecasting. The model doesn't apply to several technical features in equity market forecasting [17]. The Convolution Neural Network 
(CNN) model uses 3-dimensional tensors to combine and order the variable as an input. Then the network is trained in a way to extract useful information for market forecasting. The method developed has significant market forecasting efficiency. It was also observed that an increase of 2D-CNNpred and 3DCNNpred transaction costs accuracy decreases [18].

The economic time series prediction is implemented precisely through technical analysis by evaluating the history of stock prices and volumes. The standard annualized gain is $11.93 \%$, and the hit rate is $71.63 \%$ based on the genetic algorithm with deep multilayer perceptron (MLP) techniques. By integrating correct technical parameters and utilizing other deep neural network models, accuracy can be improved further [19]. The trends of market index prices using technical analysis and goggle trend are examined by expert system models such as ANN, SVM with different kernels. The ten technical indicators do not yield good performance; Google Trends can insufficiently predict the KOSPI 200 index price. Recurrent Neural Networks may be more accurate than the neural networks used in this study [20].

The SVM technique is proposed to forecast the movement of equity price values using various technical indicators as inputs to the models. SVM achieved a greater accuracy of $57.83 \%$ than the other models in this analysis. The accuracy of the developed model is acceptable, and the augmentation of appropriate technical indicator features improves the accuracy [21]. An optimized ANN based on a genetic algorithm (GA) developed to forecast the movement of the equity market index's price of the next day. Two types of technical indicators data sets were generated on Nikkei 225 index and added to the model proposed. They achieved $60.87 \%$ and $81.27 \%$ accuracies in the first and second data set, respectively [22].

Examined the LSTM technique was for forecasting trends in stock prices using technical analysis. The accuracy of LSTM for diverse stocks varies from 53 to $55.9 \%$. The LSTM's model has recently been developed to investigate diverse aspects of the equity market and successfully predict financial sequence data's future prices. It accomplished notably better than the baseline models. They contrasted LSTM with conventional learning tools for higher efficiency. This model given considerable returns of 0.46 percent per day [23]. Supervised artificial intelligence techniques are used to forecast the next day's direction of the stocks. Using these models, the average accuracy of the SVM for forecasting the movement of Nifty fifty stocks is $87.35 \%$, the perceptron is $75.88 \%$, and the logistic regression is $86.98 \%$. These techniques, however, are incapable of recognizing complex patterns in data during classification [24].

RNN, LSTM, and GRU deep learning models were employed to identify stock trend classification. The addition of the mechanism of the attention layer aided in improving the accuracy of the stock forecasting model. On the other hand, deeper neural networks did not always produce better results in this study [25]. Recently, researchers investigated the benefits and disadvantages of using deep learning algorithms for equity market forecasting, but their research was limited to intraday stock return forecasting [26].

The above literature reveals few deficiencies in applying technical analysis for equity market prediction. Recent studies on equity market forecasting are mostly based on their technical indicators analysis. However, numerous technical indicators exist, and applying different indicators sometimes improves forecasting accuracy. This study develops a new technical analysis technique for equity market index forecasting with deep learning to enhance deficiencies from the reviews described above.

\section{Methodology}

This paper proposes an improved predictive method for the index price movement of the equity market compared to the techniques used in the existing literature. For the prediction of index price movements, the proposed techniques use two sets of various technical indicators.

The first section presents the stock data used, and then the structure of RNN, LSTM, then explains the GRU model and the building of the three GRU variants model for bourse market prediction.

\subsection{Data collection and preparation}

Most previous scientists used numerous inputs to build prediction models. In this article, all the NIFTY 50 index data samples are collected from National Stock Exchange (NSE) provided by nseindia.com. These samples contain details from January 1, 2011, through December 31, 2019, for nine years, consisting of 2226 total samples.

The data downloaded include open, close, high, low, traded shares and turnover values. Therefore, programs from this downloaded data have to be written to calculate other technical measures to increase the accuracy of the predicted movement of stock market index prices. Table 1 presents the downloaded data description.

In this work, two sets of technical indicators are 
Table 1. Daily trading information

\begin{tabular}{|c|c|}
\hline Feature Name & Definition/Implication \\
\hline $\begin{array}{c}\text { Open and Close } \\
\text { Price }\end{array}$ & $\begin{array}{c}\text { The daily open and close price of the } \\
\text { Nifty Index }\end{array}$ \\
\hline $\begin{array}{c}\text { High and Low } \\
\text { Price }\end{array}$ & $\begin{array}{c}\text { The daily highest and lowest price } \\
\text { of the Nifty Index }\end{array}$ \\
\hline $\begin{array}{c}\text { Shares Traded } \\
\text { The Daily Traded volume of the } \\
\text { Nifty Index. }\end{array}$ \\
\hline
\end{tabular}

constructed. These indicators are measured on the basics of open, close, high, low, and share traded values; the augmented technical indicators/data used in this article and its formula revealed in Table 2 and Table 3.

\subsection{Technical data indicators}

The meaning of the technical indicators/data used in Table 2 and Table 3 are discussed here:

Simple Moving Average (SMA): A SMA is created by measuring a security's average price over a certain number of periods. In this article, 10, 20, and 50 days SMA values are used.

Exponential Moving Average (EMA): It is a form of moving average (MA) that gives the most current data points an ideal weight and implication. In this article, 10, 20, and 50 days EMA values are used.

Moving Average Convergence Divergence (MACD): The difference between two exponential moveable averages is (MACD). They are used for the buying and selling of signals.

Relative Strength Index (RSI): The RSI is used to determine whether the market has been overbought/oversold. The RSI is an overbought/oversold measure if the value is over 70 or under 30.

Stochastic Momentum Index (SMI): The SMI will compute the closing price in relative to the average of the high/low range. The SMI values vary from +100 to -100 . Extreme high / low SMI values suggest conditions that are overbought/oversold.

Relative Volatility Index (RVI): The RVI is a volatility indicator. The RVI has been used to establish the direction of uncertainty in the equity value. The RVI value ' 50 ' has been reserved as a significant point, and if the RVI above over the significant point, it enters the buy region; else, it will be in the sell region.

Ease of Movement (EMV): It exhibits the association
Table 2. Technical indicators of the NIFTY 50 index with a formula (TA Dataset 1)

\begin{tabular}{|c|c|}
\hline Indicator & Formula \\
\hline $\begin{array}{l}\text { Simple Moving } \\
\text { Average(SMA) }\end{array}$ & $\begin{array}{l}\qquad \mathrm{SMA}_{\mathrm{n}}=\frac{1}{\mathrm{n}} \sum_{\mathrm{i}=0}^{\mathrm{n}-1} \mathrm{C}_{\mathrm{t}-\mathrm{i}} \\
\text { Where Ct is a close price on day } \mathrm{t} \text {, } \\
\mathrm{n} \text { is an input window length. }\end{array}$ \\
\hline $\begin{array}{c}\text { Exponential } \\
\text { Moving } \\
\text { Average(EMA) }\end{array}$ & $\begin{array}{l}\mathrm{EMA}=(\mathrm{P} \times \alpha)+ \\
((\text { Previous EMA } \times(1-\alpha)) \\
\text { Where } \\
\mathrm{P}=\text { Current Price } \\
\alpha=\text { Smoothing Factor }=\frac{2}{1+\mathrm{N}} \\
\mathrm{N}=\text { Number of Time Periods }\end{array}$ \\
\hline $\begin{array}{l}\text { Moving } \\
\text { Average } \\
\text { Convergence } \\
\text { Divergence } \\
\text { (MACD) }\end{array}$ & $\begin{array}{l}\text { MACD = 10Peroid EMA - } \\
\text { 50Period EMA }\end{array}$ \\
\hline $\begin{array}{l}\text { Relative } \\
\text { Strength Index } \\
\text { (RSI) }\end{array}$ & $\begin{array}{l}\text { RSI }(\mathrm{N})=100-100 /(1 \\
+\operatorname{EMA}(\mathrm{N}) \text { up } \\
\qquad / \text { EMA(N)down) } \\
\text { Where EMA(N)up is upward } \\
\text { changes, EMA(N)down is } \\
\text { downward changes }\end{array}$ \\
\hline $\begin{array}{l}\text { Stochastic } \\
\text { Momentum } \\
\text { Index (SMI) }\end{array}$ & $\begin{array}{l}\quad \mathrm{SMI}=\frac{\mathrm{C}_{\mathrm{t}}-\mathrm{LL}_{\mathrm{t}-(\mathrm{n}-1)}}{\mathrm{HH}_{\mathrm{t}-(\mathrm{n}-1)}-\mathrm{LL}_{\mathrm{t}-(\mathrm{n}-1)}} \\
\qquad \times 100\end{array}$ \\
\hline $\begin{array}{c}\text { Relative } \\
\text { Volatility } \\
\text { Index (RVI) }\end{array}$ & 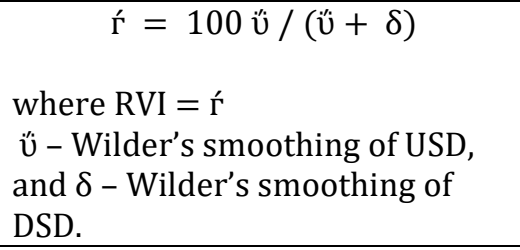 \\
\hline $\begin{array}{l}\text { Ease of } \\
\text { Movement } \\
\text { (EMV) }\end{array}$ & $\begin{array}{r}E M V=\frac{\text { Distance }}{\text { Box ratio }} \\
\text { Where } \\
\text { Distance }=\varrho=\frac{\text { High +Low }}{2}- \\
\frac{\text { Prior High+Prior Low }}{2} \\
\text { BoxRatio }=\mathrm{U}=\frac{\frac{\text { Volume }}{100,000,000}}{\text { High-Low }}\end{array}$ \\
\hline $\begin{array}{c}\text { Reynolds } \\
\text { Number( RN) }\end{array}$ & $\begin{array}{c}\mathrm{RN}=\mathrm{RVI} / \mathrm{EMV} \\
\text { Or } \\
\mathrm{R}_{\mathrm{e}}=(100 \ddot{\mathrm{v}} /(\dot{\mathrm{v}}+\delta)) /(\mathrm{Q} / \\
\mathrm{\varphi})\end{array}$ \\
\hline
\end{tabular}

between the rate of change in an asset's price and volume. When the EMV crosses above zero, a buy signal is generated, and when it crosses below zero, a sell signal is generated.

Reynolds Number (RN): In investment finance, the Reynolds number, an age-old fluid mechanics theory, 
Table 3. Technical indicators of the NIFTY 50 Index with a formula (TA Dataset 2)

\begin{tabular}{|c|c|}
\hline Indicator & Formula \\
\hline $\begin{array}{c}\text { Simple } \\
\text { Moving } \\
\text { Average(SMA) }\end{array}$ & $\begin{array}{l}\qquad \operatorname{SMA}_{\mathrm{n}}=\frac{1}{\mathrm{n}} \sum_{\mathrm{i}=0}^{\mathrm{n}-1} \mathrm{C}_{\mathrm{t}-\mathrm{i}} \\
\text { Where Ct is a close price on day } \mathrm{t} \text {, } \\
\mathrm{n} \text { is an input window length. }\end{array}$ \\
\hline $\begin{array}{c}\text { Exponential } \\
\text { Moving } \\
\text { Average(EMA } \\
\text { ) }\end{array}$ & $\begin{array}{l}\mathrm{EMA}=(\mathrm{P} \times \alpha)+ \\
((\text { Previous EMA } \times(1-\alpha)) \\
\quad \text { Where } \\
\mathrm{P}=\text { Current Price } \\
\alpha=\text { Smoothing Factor }=\frac{2}{1+\mathrm{N}} \\
\mathrm{N}=\text { Number of Time Periods }\end{array}$ \\
\hline $\begin{array}{c}\text { Moving } \\
\text { Average } \\
\text { Convergence } \\
\text { Divergence } \\
\text { (MACD) } \\
\end{array}$ & $\begin{array}{l}\text { MACD = 10Peroid EMA - } \\
\text { 50Period EMA }\end{array}$ \\
\hline $\begin{array}{c}\text { Relative } \\
\text { Strength Index } \\
\text { (RSI) }\end{array}$ & $\begin{array}{l}\text { RSI(N)=100- } 100 /(1 \\
+\operatorname{EMA}(\mathrm{N}) \text { up } \\
\qquad / \text { EMA(N)down }) \\
\text { Where EMA(N)up is upward } \\
\text { changes, EMA(N)down is } \\
\text { downward changes }\end{array}$ \\
\hline
\end{tabular}

has been redefined to classify potential explosive moments in the stock market.

\subsection{Recurrent neural networks}

A standard time series data is stock index data. The GRU variant model with enhanced technical analysis is used to handle forecasting stock index value. In this section, the first RNN and LSTM structures are discussed. Finally, proposed three variants of GRU, namely GRU1, GRU2, and GRU3, are discussed.

RNN: The RNN is a type of ANN implemented to model the time series in the 1980s. The core idea with which the RNN was developed is to use a memory. This memory holds the data from the preprocessing steps. This information plays an essential role in predicting the following steps accurately [27]. The algorithm used in RNN is backpropagation through time (BPTT); this algorithm is generally used in ANN to determine a gradient and update the network's weights. The prediction of RNN is given by Eq. (1).

$$
h_{t}=f\left(X_{t}, h_{t-1}\right)
$$

Where ht- 1 is the hidden state in the previous time steps of the (t-1)-th, and Xt is the current input.
LSTM: To resolve the "vanishing" or "exploding" gradient exertion, the disappearance of gradients, two basic techniques, the LSTM unit and GRU, were suggested [27]. The efficiency of LSTM networks trained by backpropagation through time for equity market forecasting is analyzed [28].

An LSTM can replace the hidden units in the memory blocks of RNN, and it has three main gates, known as input gate, output gate, and forget gate. The state of the retention degree at the last moments of the cell is determined by forget gate. The input gate identifies the quantum of the network's input, which is saved to the cell state at the current time. According to the LSTM output, the output gate's unit state output is controlled [29]. All of the relationships are definite, as shown in Eqs. (2), (3), (4), (5), (6), and (7).

$$
\begin{aligned}
& f_{t}=\sigma\left(W_{f}\left[h_{t-1}, x_{t}\right]+b_{f}\right. \\
& i_{t}=\sigma\left(W_{i}\left[h_{t-1}, x_{t}\right]+b_{i}\right. \\
& C_{t}^{\prime}=\tanh \left(W_{C}\left[h_{t-1}, x_{t}\right]+b_{C}\right) \\
& C_{t}=f_{t} * C_{t-1}+i_{t} * C_{t}^{\prime} \\
& o_{t}=\sigma\left(W_{o}\left[h_{t-1}, x_{t}\right]+b_{o}\right) \\
& h_{t}=o_{t}+\tanh \left(C_{t}\right)
\end{aligned}
$$

Where $\mathrm{W}$ represents the matrix of weight and $\mathrm{b}$ is $\mathrm{a}$ vector of bias. Furthermore, at time step $t, x t$ is the input vector, ht is the output vector, and at time step $\mathrm{t}, \sigma$ is a sigmoid function, and $\mathrm{Ct}$ is the cell state.

GRU: Stock market forecasting based on deep learning provides considerable performance. Deep learning methods used in stock market forecasting helps in the useful analysis of nonlinear factors. LSTM networks with a slight variation can be known as GRU. However, the LSTM has several parameters, and it takes additional time to transform the model's

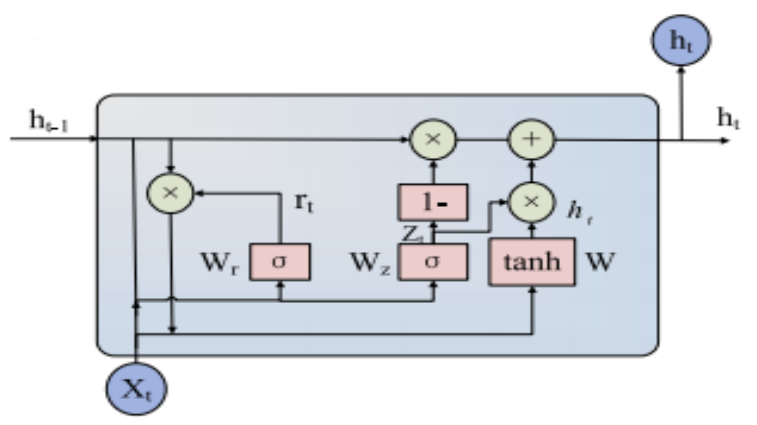

Figure. 1 The GRU module structure 
parameters to be the finest. Both LSTM and GRU produce excellent results in some cases [30]. Along with the LSTM unit, GRU is a primarily suggested technique for sequence data issues in deep learning. As in LSTM cell architecture, a separated memory cell is not available to GRU cells. Unlike LSTM, which has three gating layers, only two gating layers are available in GRU, namely update gate $\left(Z_{t}\right)$ and reset gate $\left(r_{t}\right)$. GRU is used to determine the quantum of previous information memory, which can be stored for the future [31]. The GRU gate equations shown in Eqs. (8), (9), (10), and (11). The structure of a GRU module is seen in Fig. 1

$$
\begin{aligned}
& Z_{t}=\sigma\left(W_{z} h_{t-1}+W_{z} x_{t}+b_{z}\right) \\
& r_{t}=\sigma\left(W_{r} h_{t-1}+W_{r} x_{t}+b_{r}\right) \\
& h_{t}^{\prime}=\tanh \left(W_{r}\left(h_{t-1} r_{t}\right)+W_{f} x_{t}+b_{h}\right) \\
& h_{t}=\left(1-z_{t}\right) h_{t-1}+Z_{t} h_{t}^{\prime}
\end{aligned}
$$

Where $x t$ is the input vector at time step $t$, ht is the output vector at time step $\mathrm{t}, \mathrm{W}$ represents the matrix of weight, $b$ is a vector of bias, and $\sigma$ is a sigmoid function.

\subsection{Proposed variant GRU models}

This article concentrates on the GRU RNN and investigates three gate-variants with decreased parameterization on financial time series forecasting. A Gated Recurrent Unit (GRU) was suggested to allow the recurrent unit to capture the long-term dependencies of various time scales dynamically. Using the backpropagation by time (BTT) stochastic gradient descent, the weights equivalent to these gates are often updated as they attempt to minimize the error. The three variants of GRU differ in the process of previous hidden states and bias [32]. This article proposed model uses three distinct variants of the GRU RNN are implemented by dropping parameters in the update and reset gates gating equations by combining the technical analysis.

Variant 1 - GRU1: In this model, the previous hidden state and the bias were used to measure each gate shown in Eqs. (12) and (13). The total number of parameters thus decreased by $2 \mathrm{~nm}$ relative to the GRU RNN.

$$
\begin{aligned}
& z_{t}=\sigma\left(U_{z} h_{t-1}+b_{z}\right) \\
& r_{t}=\sigma\left(U_{r} h_{t-1}+b_{r}\right)
\end{aligned}
$$

Variant 2 - GRU2: In this model, the previous hidden state is used for the calculation of each gate shown in Eqs.(14) and (15). The total number of parameters thus decreased by two times $(n m+n)$ relative to the GRU RNN.

$$
\begin{aligned}
& z_{t}=\sigma\left(U_{z} h_{t-1}\right) \\
& r_{t}=\sigma\left(U_{r} h_{t-1}\right)
\end{aligned}
$$

Variant 3 - GRU3: In this model, only bias is used to compute each gate shown in Eqs.(16) and (17). The total number of parameters is thus decreased by two times $(n m+n 2)$ relative to the GRU RNN.

$$
\begin{aligned}
& z_{t}=\sigma\left(b_{z}\right) \\
& r_{t}=\sigma\left(b_{r}\right)
\end{aligned}
$$

In this research article, in contrast to the RNN, LSTM models, we conducted an empirical analysis of the results of each of these three variants on two technical predictor datasets, namely TA dataset1 (Table 2.) and TA dataset2 (Table 3.).

\subsection{Proposed architecture}

In this section, we discuss the proposed architecture of the stock forecasting model using GRU variants. In this architecture, from the NIFTY 50 index OHLC and volume data, the technical indicators (feature extraction) were calculated. These

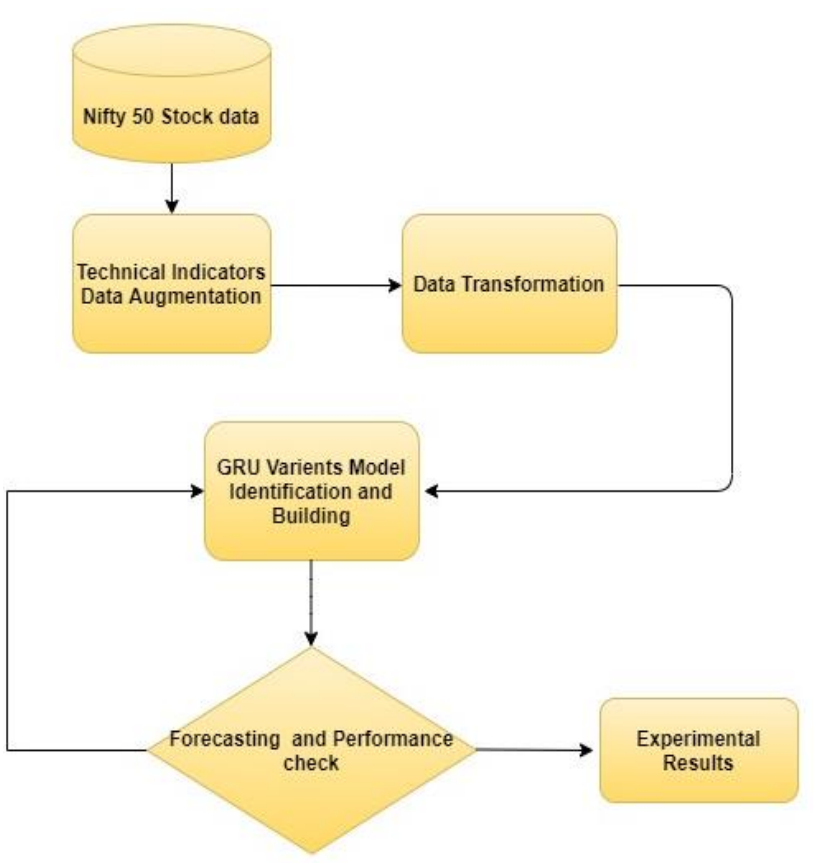

Figure. 2 Stock forecasting model using GRU variants model 
are normalized using the normalization technique and fed into to the proposed three GRU variants method. For predicting the NIFTY 50 index price movement, the proposed three GRU variants analysis uses a diverse set of technical indicators (technical data augmentation). The proposed stock forecasting framework is show in Fig. 2.

We performed an experiential analysis of the three distinct derived GRU equations in this study with two datasets of technical metrics, namely TA dataset1 (Table 2.) and TA dataset2 (Table 3.), and compared to the RNN, LSTM models to validate our analysis. The experimental results indicate that TA dataset 1 outperformed the proposed three GRU variants compared to TA dataset 2 . In this research, the GRU unit variants are developed in Python code in the Keras library with a back-end library as a Theano in the google colab environment. These GRU variants are tested with Rectified Linear Unit ReLU) activation function, batch size of 64, Time steps of 10, Adam Optimizer, 100 Epochs, and Dropout 20\% deep learning hyper parameters.

\section{Experimental results and discussion}

This section examines the results obtained from the experiments carried out during the implementation of this study. Deep neural networks provide various distinct advantages than conventional models: self-learning, non-assumption, noise-tolerant, and capturing nonlinear interdependence that is not typical to traditional models [33-34]. Therefore, deep neural networks are typically more efficient than conventional models in forecasting stock index prices [35]. The aim is to forecast the NIFTY 50 index price movement using reduced GRU variants deep learning algorithms and then compare these algorithms's performance with RNN and LSTM models.

The data for nine years, consisting of 2226 total samples, was used from January 1, 2011, until December 31, 2019. The dataset for training and testing consists of 1781 and 445 samples. The three variants of GRU are evaluated in the dataset of the NIFTY 50 index by performing technical data augmentation. To examine performance, the proposed GRU variants (V1, V2, and V3) approach are compared with the current deep learning methods of RNN and LSTM. The proposed and the existing methods have been tested in the same framework environment. The experiments are evaluated in the Goggle Colab environment system. The hyperparameters settings of the RNN, LSTM and GRU variants models are epochs set as 100, and a

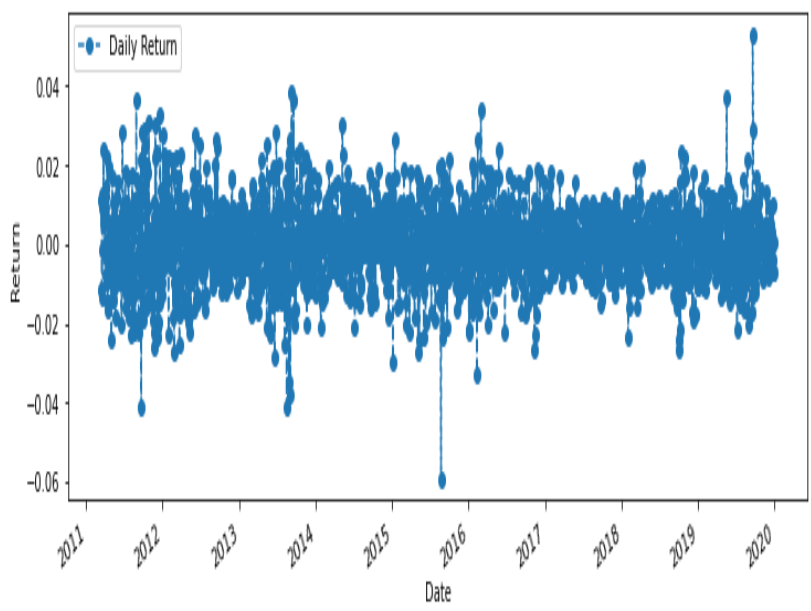

Figure. 3 Nifty 50 index daily return.

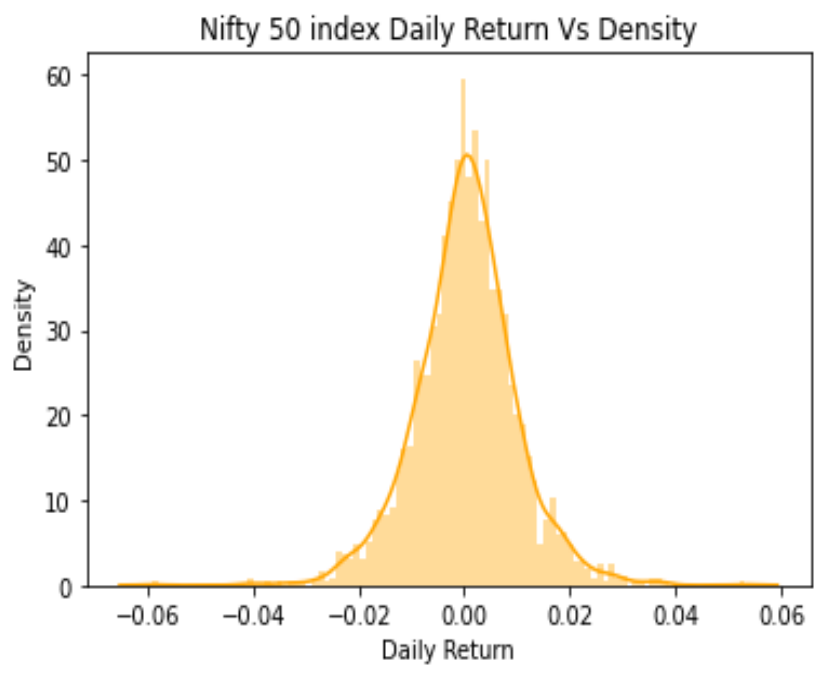

Figure. 4 Nifty 50 index daily return vs. density

dropout range is set as 0.2 , the batch size is set as 64 , time steps 30, and optimizer developed as Adam. Following Fig. 3 reveals the regular return of the NIFTY 50 index from January 2011 to December 2019. In the year 2019 NIFTY 50 index has given a high return of 0.04 . Fig. 4 shows NIFTY 50 index daily return vs. density.

A comparison was conducted between two technical indicator datasets by training RNN, LSTM, and three GRU variants to authenticate the proposed technical stock data augmentation method. The error value is measured between the proposed and existing methods and compared to analyze the performance. In this study, to assess the effectiveness of the proposed deep learning models, the MAE, MSE, and RMSE metrics were measured and established in Eqs. (18), (19), and (20). 
Table 4. Performance comparison of RNN, LSTM, and three variants of GRU models on two types of technical features (namely TA1 and TA2) of the Nifty 50 index

\begin{tabular}{|c|c|c|c|c|c|c|c|c|c|c|c|c|}
\hline \multirow{3}{*}{ Method } & \multicolumn{6}{|c|}{ TA Dataset1 } & \multicolumn{6}{|c|}{ TA Dataset2 } \\
\hline & \multicolumn{3}{|c|}{ Training Results } & \multicolumn{3}{|c|}{ Testing Results } & \multicolumn{3}{|c|}{ Training Results } & \multicolumn{3}{|c|}{ Testing Results } \\
\hline & MSE & MAE & RMSE & MSE & MAE & RMSE & MSE & MAE & RMSE & MSE & MAE & RMSE \\
\hline $\begin{array}{c}\text { Simple } \\
\text { RNN }\end{array}$ & 0.241 & 0.429 & 0.491 & 1.277 & 1.111 & 1.130 & 0.270 & 0.439 & 0.519 & 1.245 & 1.024 & 1.115 \\
\hline LSTM & 0.006 & 0.060 & 0.078 & 0.183 & 0.394 & 0.428 & 0.025 & 0.119 & 0.159 & 0.463 & 0.659 & 0.681 \\
\hline GRU 1 & 0.001 & 0.025 & 0.034 & 0.062 & 0.215 & 0.249 & 0.023 & 0.109 & 0.152 & 0.431 & 0.626 & 0.656 \\
\hline GRU 2 & 0.003 & 0.041 & 0.057 & 0.101 & 0.289 & 0.319 & 0.025 & 0.105 & 0.158 & 0.559 & 0.731 & 0.747 \\
\hline GRU 3 & 0.002 & 0.039 & 0.048 & 0.014 & 0.106 & 0.120 & 0.034 & 0.130 & 0.185 & 0.585 & 0.746 & 0.765 \\
\hline
\end{tabular}
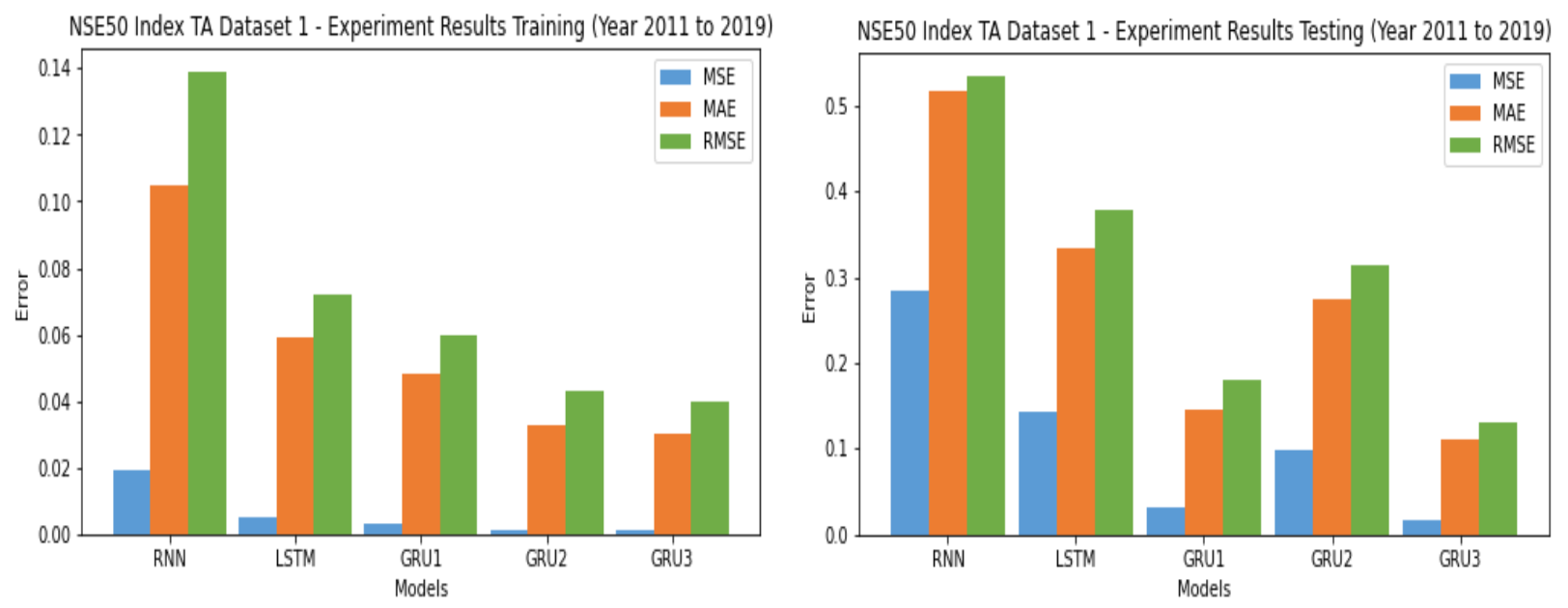

Figure. 5 Training and testing error value analysis on Nifty 50 index using RNN, LSTM, and three variants of GRU using TA dataset 1
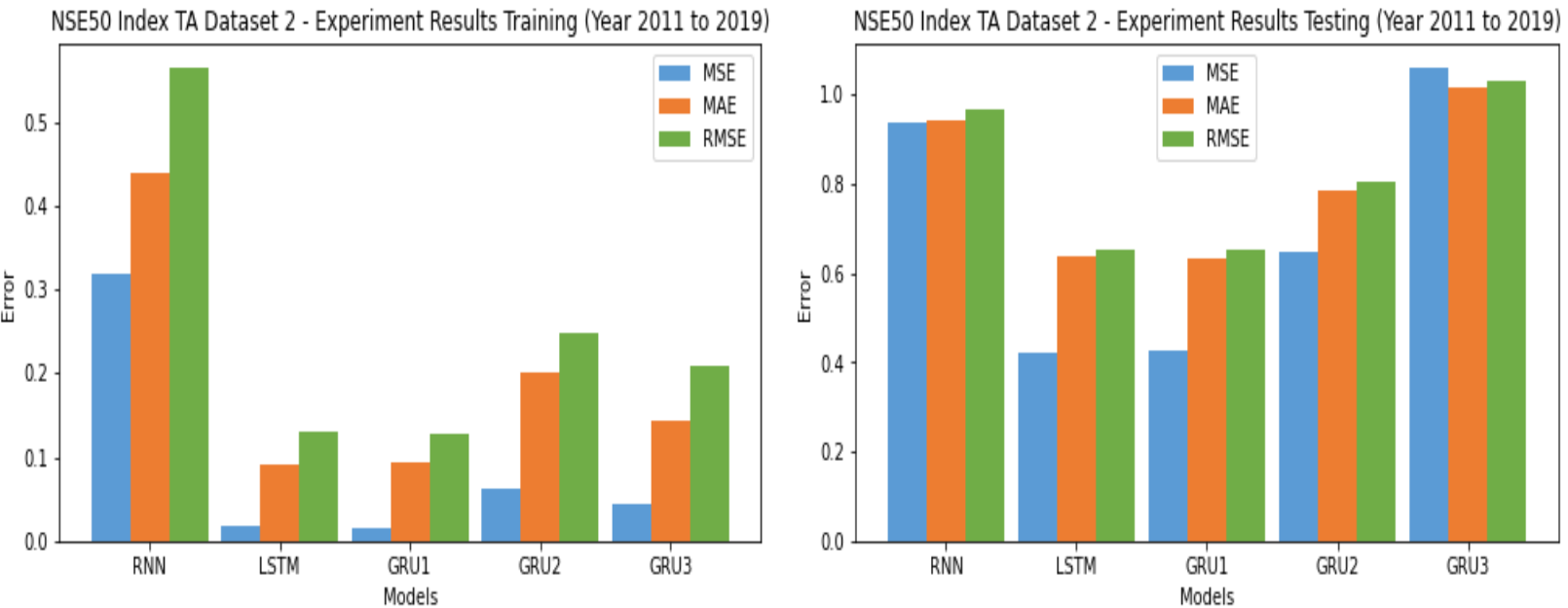

Figure. 6 Training and testing error value analysis on Nifty 50 index using RNN, LSTM, and three variants of GRU using TA dataset 2 


$$
\begin{aligned}
& M S E=\frac{1}{n} \sum_{i=1}^{n}\left(Y_{i}-Y_{\text {hat }}\right)^{2} \\
& M A E=\frac{1}{n} \sum_{i=1}^{n}\left|Y_{i}-Y_{\text {hat }}\right| \\
& R M S E=\sqrt{\sum_{i=1}^{n} \frac{\left(Y_{i}-Y_{\text {hat }}\right)^{2}}{n}}
\end{aligned}
$$

The error value of MSE, RMSE and MAE is measured for the proposed and existing method in the Nifty 50 index dataset. The error values are calculated for the proposed GRU and the current RNN and LSTM models using the technical indicators of Table 2 (i.e., TA Dataset1) and technical indicators of Table 3 (i.e., TA dataset 2) in above Table 4 . The proposed GRU's error value and the existing RNN and LSTM models are compared in Fig. 5 and Fig. 6. Fig. 5 shows the RNN, LSTM, and three GRU variants, training, and testing error measurement analysis on the Nifty 50 index using technical indicators from Table 2. The RNN, LSTM, and three GRU versions, training, and test error calculation analysis on the Nifty 50 index are shown in Fig. 6 using the technical indicators from Table 3.

The results from above Fig. 5 and Fig. 6 shows that the developed GRU method has higher performance(lower error rate) than RNN and LSTM using technical indicators from Table 2 (TA dataset 1) compared to technical indicators from Table 3 (TA dataset 2). The LSTM method has a considerable performance in forecasting due to its capacity to store information in the long term. The RNN method has a higher error value due to the vanishing gradient problem. The GRU method's a simpler version of LSTM solves the vanishing gradient problem, which has a lower error value than other methods on technical indicators from Table 2(i.e., TA dataset 1).

The technical indicators set 1 from Table 2 (i.e., TA dataset 1), which uses SMA, EMA, MACD,

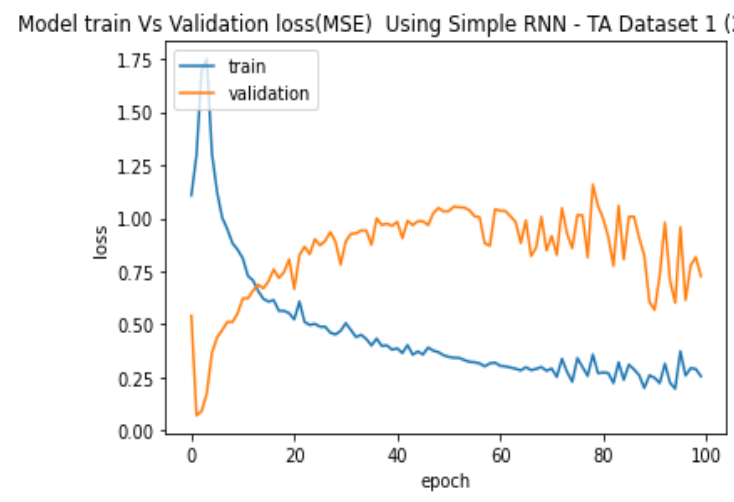

(a)

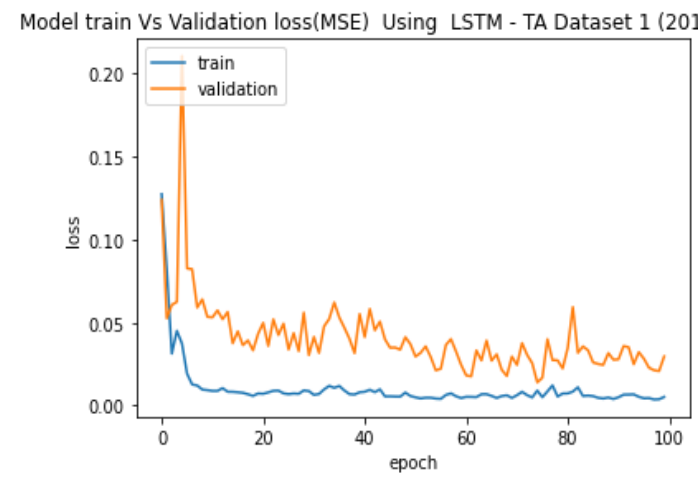

(b)

Figure. 7 Results of RNN and LSTM model using technical indicators of Table 2(TA dataset 1):

(a) RNN Model loss on the Nifty 50 index (b) LSTM Model loss on Nifty 50 index

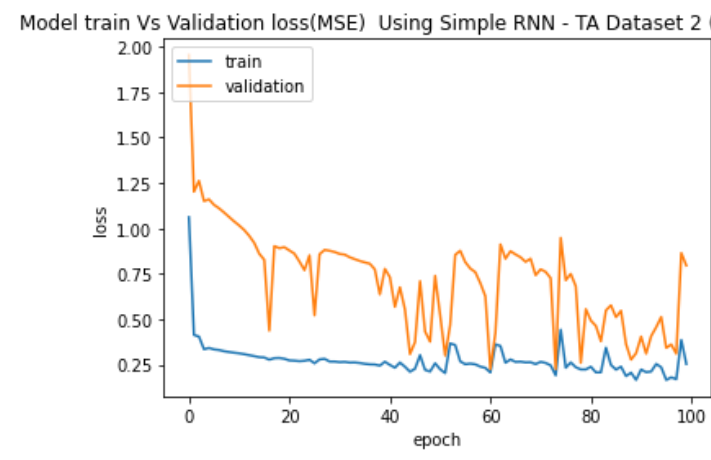

(a)

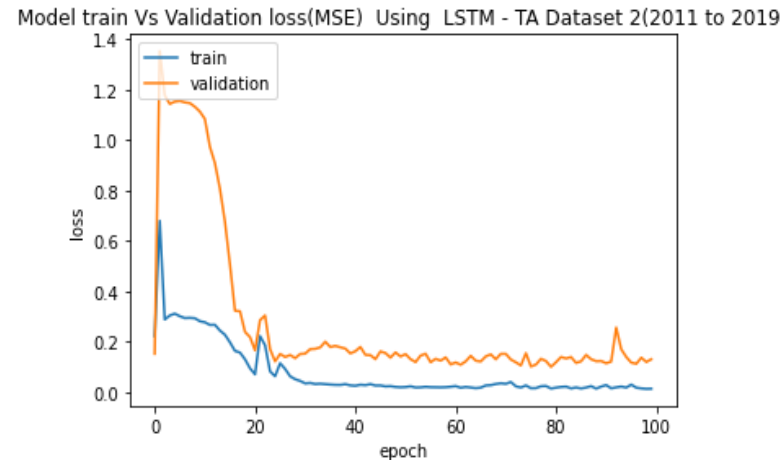

(b)

Figure. 8 Results of RNN and LSTM model using technical indicators of Table 3(TA dataset 2):

(a) RNN Model loss on the Nifty 50 index (b) LSTM Model loss on Nifty 50 index 
Model train Vs Validation loss(MSE) Using GRU Varient 1 - TA Dataset 1 (2011 to 2019)

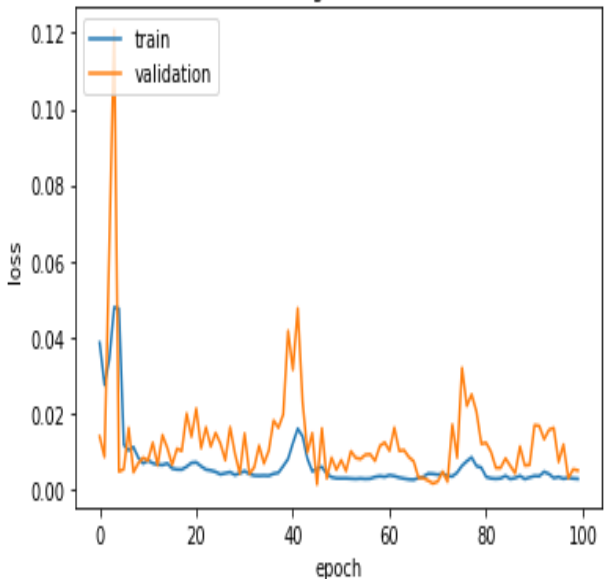

(a)

Model train Vs Validation loss(MSE) Using GRU Varient 3 - TA Dataset 1 (2011 to 2019)

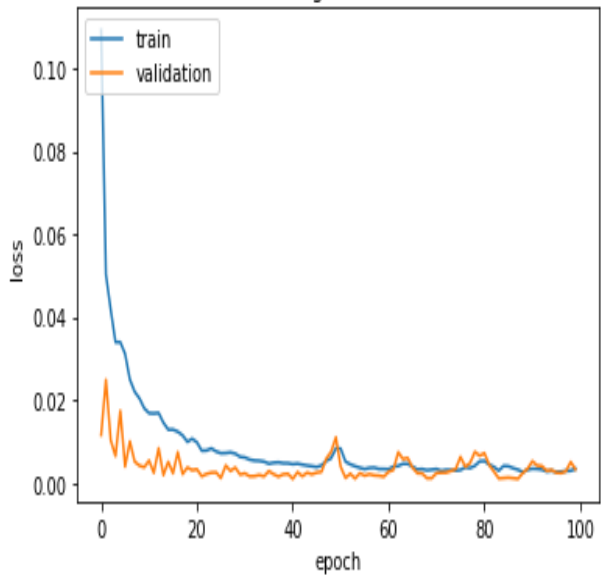

(c)
Model train Vs Validation loss(MSE) Using GRU Varient 2 - TA Dataset 1 (2011 to 2019)

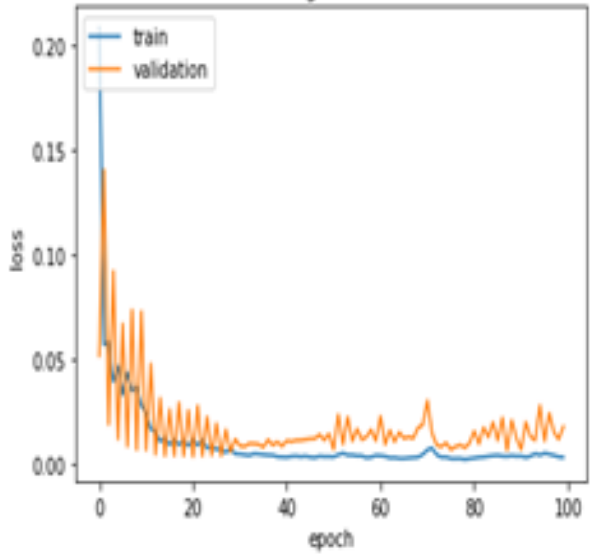

(b)

Model train Vs Validation loss(MSE) Using GRU Varient 1 - TA Dataset 2 (2011 to 2019)

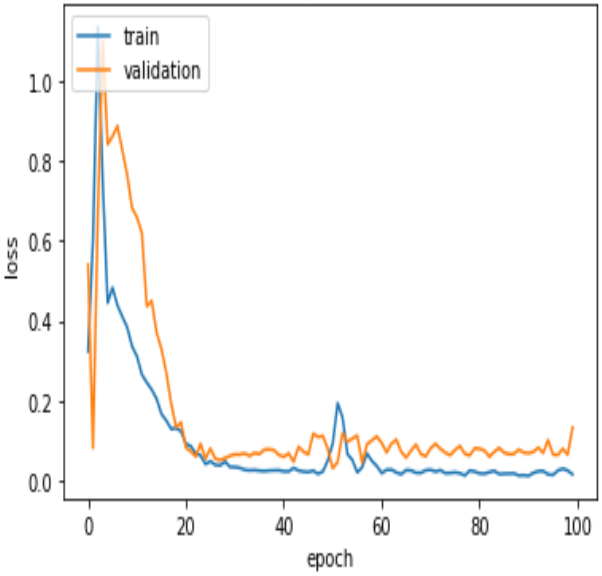

(d)

Model train Vs Validation loss(MSE) Using GRU Varient 2 - TA Dataset 2 (2011 to 201

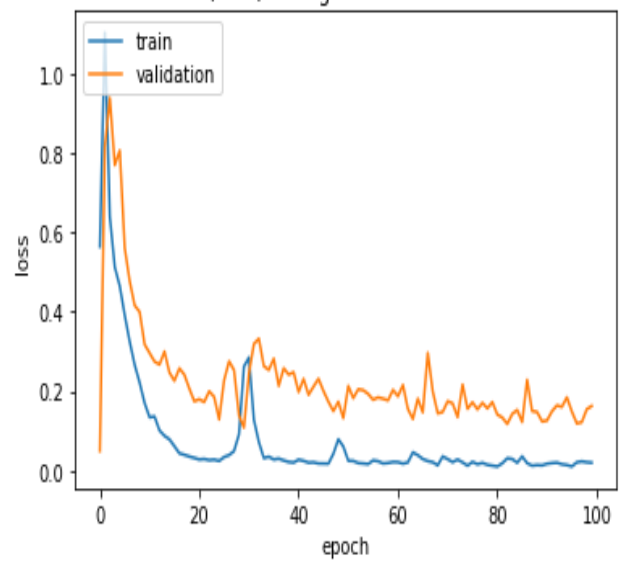

(e)

Model train Vs Validation loss(MSE) Using GRU Varient 3 - TA Dataset 2 (2011 to 2019)

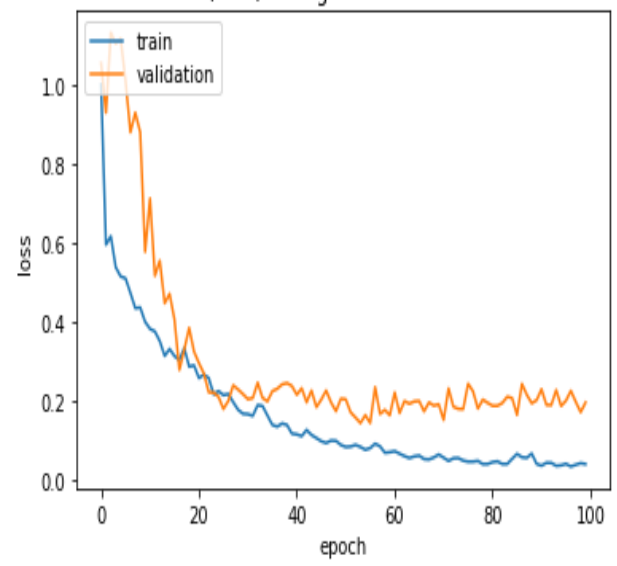

(f)

Figure. 9 Performances of three variants of GRU model on Nifty 50 index using technical analysis: (a), (b) and (c) Three variants of GRU model loss using technical indicators of Table 2(TA dataset 1) (d),(e) and (f) Three variants of GRU model loss using technical indicators of Table 3(TA dataset 2)

RSI indicators along with additional Relative Volatility Index(RVI), Reynolds Number(RN), and Ease of movement (EMV) indicators. The computation of the relative volatility index (RVI) and ease of movement (EMV) is done to confirm the financial version of Reynolds Number (RN) [13]. The 
technical indicators of Table 3 (i.e., TA dataset 2) use SMA, EMA, MACD, and RSI indicators. However, the experimental results from above Fig. 6 show that the developed RNN, LSTM, and GRU methods have lower performance (high error rate), which uses SMA, EMA, MACD, and RSI technical indicators.

Fig. 7 depicts the RNN and LSTM model loss on the Nifty 50 index using technical indicators of Table 2(TA dataset1). Fig. 8 depicts the RNN and LSTM model loss on the Nifty 50 index using technical indicators of Table 3(TA dataset 2). The test results from Fig. 7 and Fig. 8 indicate that methods for RNN and LSTM use technical indicator dataset 2 (TA dataset2) to achieve lower performance than technical indicator data set 1 (TA dataset1).

Fig. 9 shows the performance of three variants of GRU model loss on the Nifty 50 index using the technical indicators of Table 2 (i.e., TA Dataset1) and technical indicators of Table 3 (i.e., TA dataset 2). The experimental outcome shows that the developed GRU variants method has higher performance (lower error rate) using technical indicator dataset 1 (TA dataset1) compared to RNN and LSTM models.

The experimental results and discussion from this section show that the developed GRU variants have a higher performance than other existing methods of LSTM and RNN. These proposed three variants of GRU will derive additional conceptual and invariant features. Therefore the GRU neural network's gate variants based on the deep learning model predict India NIFTY 50 index price movement by performing a technical data augmentation approach, enhancing the model's overall predictive capability.

\section{Conclusion and future work}

This study has proposed a model that combines technical analysis with three variants of GRU deep learning approaches to predict equity price movements on the NIFTY 50 index efficiently. It can reap financial benefits for investors who trade based on the future price prediction. In this work, nine years of historical stock data were used, and the computation of two sets of technical indicators for the training and learning of deep learning models. The developed GRU variants are analyzed on two sets of technical indicators (namely TA1 or TA2) are used, and compared to the RNN and LSTM models.

The main findings and contributions of this study are as follows:

- Based on technical analysis, this study developed an equity market forecasting process.

- The performance of RNN, LSTM, and three variants of GRU models were compared on two technical feature sets (namely, TA1 and TA2) of the NIFTY 50 index. GRU has found TA1 features to be more efficient based on experimental results. TA1 features may therefore be used to build a practical modeling approach for predicting index price movement.

- The technical indicators such as Stochastic Momentum Index (SMI) and Reynolds Number $(\mathrm{RN})$ carry more information about index price movement than others.

- The predictive power of deep learning models can reap financial benefits for investors who trade based on the future price prediction.

- The proposed technique in this work provides a valuable model of reference that can be used with other financial instruments such as bonds, foreign exchange rates, crypto currencies, and mutual funds.

\section{Conflicts of interest}

The authors declare no conflict of interest.

\section{Author contributions}

In this research article author contribution are as follows: "Conceptualization and Methodology by Balamurugan Marimuthu, Bikramaditya Ghosh, and Chinthakunta Manjunath; Implementation by Chinthakunta Manjunath; Writing - original draft preparation by Chinthakunta Manjunath; Writingreview and editing by Balamurugan Marimuthu, and Bikramaditya Ghosh".

\section{Acknowledgments}

We are grateful for the technological infrastructure help CHRIST (Deemed to be University) Bangalore, India, to do our research work.

\section{References}

[1] Y. S. A. Mostafa and A. F. Atiya, "Introduction to Financial Forecasting", Applied Intelligence, Vol. 6, No. 3, pp. 205-213, 1996.

[2] C. Huang and J. Cao, "Active Control Strategy for Synchronization and Anti-Synchronization of a Fractional Chaotic Financial System", Physica A: Statistical Mechanics and its Applications, Vol. 473, pp. 262-275, 2017.

[3] E. Chong, C. Han, and F. C. Park, "Deep Learning Networks for Stock Market Analysis and Prediction: Methodology, Data Representations, and Case Studies", Expert Systems with Applications, Vol. 83, pp. 187-205, 2017. 
[4] H. Wang, L. Shan, and J. Zhao, "Aggregating Multiple types of Complex Data in Stock Market Prediction: A Model-Independent Framework", Knowledge Based Systems, Vol. 164, pp.193204, 2019.

[5] X. Zhang, Y. Zhang, S. Wang, Y. Yao, B. Fang, and $\mathrm{P}$. $\mathrm{S} \mathrm{Yu}$, "Improving Stock Market Prediction via Heterogeneous Information Fusion", Knowledge Based Systems, Vol. 143, pp. 236-247, 2018.

[6] X. Zhang, J. Shi, D. Wang, and B. Fang, "Exploiting Investors Social Network for Stock Prediction in China's Market", Journal of Computational Science, Vol. 28, pp. 294-303, 2018.

[7] K. Nam and N. Seong, "Financial News-Based Stock Movement Prediction Using Causality Analysis of Influence in the Korean Stock Market", Decision Support Systems, Vol. 117, pp. 100-112, 2019.

[8] N. Oliveira, P. Cortez, and N. Areal, "The Impact of Micro Blogging Data for Stock Market Prediction: Using Twitter to Predict Returns, Volatility, Trading volume, and Survey Sentiment Indices", Expert Systems with Applications, Vol. 73, pp. 125-144, 2017.

[9] M. Qiu, Y. Song, and F. Akagi, "Application of Artificial Neural Network for the Prediction of Stock Market Returns: The case of the Japanese Stock Market", Chaos, Solitons \& Fractals, Vol. 85, pp.1-7, 2016.

[10] S. P Chatzis, V. Siakoulis, A. Petropoulos, E. Stavroulakis, and N. Vlachogiannakis, "Forecasting Stock Market Crisis Events using Deep and Statistical Machine Learning Techniques", Expert Systems with Applications, Vol. 112, pp. 353-371, 2018.

[11] W. Long, Z. Lu, and L. Cui, "Deep LearningBased Feature Engineering for Stock Price Movement Prediction", Knowledge-Based Systems, Vol. 164, pp. 163-173, 2019.

[12] B. Ghosh, "Quest for Behavioural Traces the Neural Way : A Study on BSE 100 along with its Oscillators", Indian Journal of Research in Capital Markets, Vol. 4,pp. 19-25, 2017.

[13] B. Ghosh, "Comparative Predictive Modeling on CNX Nifty with Artificial Neural Network", SDMIMD Journal of Management, Vol. 7, pp. 1-7, 2016.

[14] Y. Shynkevich, T. M. McGinnity, S. A. Coleman, A. Belatreche, and Y. Li, "Forecasting Price Movements using Technical Indicators: Investigating the Impact of Varying Input Window Length", Neurocomputing, Vol. 264, pp. 71-88, 2017.
[15] J. Patel, S. Shah, P. Thakkar, and K. Kotecha, "Predicting Stock and Stock price Index Movement Using Trend Deterministic Data Preparation and Machine Learning Technique", Expert Systems with Applications, Vol. 42, No. 1, pp. 259-268, 2015.

[16] B. Ghosh and Emira K, "Identifying Explosive behavioral Trace in the CNX Nifty Index: A Quantum Finance Approach", Investment Management and Financial Innovations, Vol. 15 (1), pp. 208-223, 2018.

[17] Y. Baek and H. Y. Kim, "ModAugNet: A New Forecasting Framework for Stock Market Index Value with an overfitting Prevention LSTM module and a Prediction LSTM module", Expert Systems with Applications, Vol. 113, pp. 457480, 2018.

[18] E. Hoseinzade and S. Haratizadeh, "CNNpred: CNN-based Stock Market Prediction using a Diverse Set of Variables", Expert Systems with Applications, Vol. 129, pp. 273-285, 2019.

[19] O. B. Sezer, M. Ozbayoglu, and E. Dogdu, "A Deep Neural-Network Based Stock Trading System Based on Evolutionary Optimized Technical Analysis Parameters", Procedia Computer Science, Vol. 114, pp. 473-480, 2017.

[20] S. Pyo, J. Lee, M. Cha, and H. Jang, "Predictability of Machine Learning Techniques to Forecast the Trends of Market Index Prices: Hypothesis Testing for the Korean Stock Markets", PLoS One, Vol. 12, No. 11, 2017.

[21] B. Gui, X. Wei, Q. Shen, J. Qi, and L. Guo, "Financial Time Series Forecasting using Support Vector Machine", In: Proc. of 10th International Conf. on Computational Intelligence and Security, CIS 2014, pp. 39-43, 2015.

[22] M. Qiu and Y. Song, "Predicting the Direction of Stock Market Index Movement using An Optimized Artificial Neural Network Model", PLOS ONE, Vol. 11, 2016.

[23] D. M. Q. Nelson, A. C. M. Pereira, and R. A. D Oliveira, "Stock Market's Price Movement Prediction with LSTM Neural Networks", International Joint Conference on Neural Networks, pp. 1419-1426, 2017.

[24] I. Ramzan, P. Surinder, S. Khurana, M. Kumar, and A. A. Altalbe, "Time Series Data Analysis of Stock Price Movement using Machine Learning Techniques", Soft Computing, Vol. 24, No. 21, pp. 16509-16517, 2020.

[25] J. Zhao, D. Zeng, S. Liang, H. Kang, and Q. Liu, "Prediction Model for Stock Price Trend Based on Recurrent Neural Network", Journal of Ambient Intelligence and Humanized 
Computing, Vol. 12, No. 1, pp. 745-753, 2020.

[26] E. Chong, C. Han, and F. C. Park, "Deep Learning Networks for Stock Market Analysis and Prediction: Methodology, Data Representations, and Case Studies", Expert Systems with Applications, Vol. 83, pp. 187-205, 2017.

[27] S. Hochreiter and J. Schmidhuber, "Long ShortTerm Memory", Neural Computation, Vol. 9, No 8, pp.01735-1780, 1997.

[28] H. Jia, "Investigation into the Effectiveness of Long Short Term Memory Networks for Stock Price Prediction", arXiv [cs.NE], pp. 1-6, 2016.

[29] W. Bao, J. Yue, and Y. Rao, "A deep Learning Framework for Financial Time Series using Stacked Auto Encoders and Long-Short Term Memory", PloS One, Vol. 12, No. 7, 2017.

[30] W. Jiang, "Applications of Deep Learning in Stock Market Prediction: Recent Progress", ArXiv, abs/2003. 01859, 2020.

[31] K. A. Althelaya, E. M. E. Alfy, and S. Mohammed, "Stock Market Forecast Using Multivariate Analysis with Bidirectional and Stacked (LSTM, GRU)", In: Porc. of 21st Saudi Computer Society National Computer Conference (NCC), pp. 1-7, 2018.

[32] R. Dey and F. M. Salem, "Gate-variants of Gated Recurrent Unit (GRU) Neural Networks", In: Proc. of IEEE 60th International Midwest Symposium on Circuits and Systems (MWSCAS), Boston, MA, pp. 1597-1600, 2017.

[33] L. D. Persio and O. Honchar, "Artificial Neural Networks Approach to the Forecast of Stock Market Price Movements", International Journal of Economics and Management Systems, Vol. 1, pp. 158-162, 2016.

[34] H. Yan and H. Ouyang, "Financial Time Series Prediction based on Deep Learning", Wireless Personal Communications, Vol. 102, No. 2, pp. 683-700, 2018.

[35] J. Henríquez and W. Kristjanpoller, "A Combined Independent Component Analysis Neural Network Model for Forecasting Exchange Rate Variation", Applied Soft Computing, Vol. 83, 2019. 